Dysphagia 12:171 (1997)

\title{
Dysphagia
}

(c) Springer-Verlag New York Inc. 1997

Letter to the Editor

\section{Function of the Inferior Pharyngeal Constrictor Muscle}

Although the effects of the pharyngeal constrictors were well documented by Olsson et al. (Dysphagia 11: 83-86, 1996), little is known about the cause of different mechanical constraints at different levels of the pharynx [1].

Olsson et al. stated that the pressure rise was more than twice as high at the inferior pharyngeal constrictor level than at the middle pharyngeal constrictor level, and pressure recordings within the pharynx depend very much on axial and longitudinal asymmetry.

We found recently the three branches of the superior laryngeal nerve (SLN) in the rat [2]. The internal branch of the SLN corresponded to the rostral branch, and the external branch corresponded to the middle branch and the caudal branch. Afferent fibers were evident in the rostral branch and caudal branch, and efferent fibers were in the middle branch and caudal branch. The rostral branch seemed to be sensitive for elicitation of the swallowing reflex. The middle branch innervated the cricothyroid muscle, which does not exhibit a stretch reflex. Labeled cells were observed within the ambiguus nucleus and in the area ventrolateral to the ambiguus, which is referred to as the rostral ventral respiration group, following application of HRP-WGA to the middle branch. Caudal branch enters the inferior pharyngeal constrictor muscle, and proprioceptive units were recorded from the peripheral cut end of the caudal branch. The afferent projections of the caudal branch were observed in the nucleus of the solitary tract. Efferent discharges from the caudal branch exhibited irregular bursts not synchronized with respiration. These motoneurons were easily affected by the depth of anesthesia and animal's condition. HRP labeled cells were observed within both the ambiguus nucleus and the dorsal motor nucleus following the application of HRP-WGA to the caudal branch.
Our study indicates clear differences in physiological activity and central distribution of the three branches of the SLN. The detection of proprioceptive units in the inferior pharyngeal constrictor muscle has important clinical implications, since the movement of lower pharynx may be tightly regulated by their units, and a cause of aspiration may be in part attributed to failure of prompt recognition and action in the inferior pharyngeal constrictor muscle as well as failure to respond rapidly to the bolus in the region innervated by the internal branch. Thus, the SLN may form a major component of swallowing. Although the species were different between the study by Olsson et al. and us, our findings support that videomanometry may be useful in diagnosis of disorder of the inferior pharyngeal constrictor muscle. In addition, since caudal branch is invisible to the surgeon at the level of the inferior pharyngeal constrictor muscle, trachea, pharynx, and the carotid sheath should be carefully prepared.

Minoru Yamaoka, D.D.S., Ph.D. Kiyofumi Furusawa, D.D.S., Ph.D. Oral and Maxillofacial Surgery Department II Matsumoto Dental College Shiojiri, Nagano, Japan

\section{References}

1. Olsson R, Kjellin O, Ekberg $\mathrm{O}$ : Videomanometric aspects of pharyngeal constrictor activity. Dysphagia 11:83-86, 1996

2. Furusawa K, Yasuda K, Okuda D, Tanaka M, Yamaoka M: Central distribution and peripheral functional properties of afferent and efferent components of the superior laryngeal nerve: Morphological and electrophysiological studies in the rat. $J$. Comp. Neurol. 375:147-159, 1996 